\title{
ESTUDO DESCRITIVO DA QUALIDADE DA INFORMAÇÃO NO PLANEJAMENTO DA PRODUÇÃO
}

\section{DESCRIPTIVE ANALYSIS OF PRODUCTION PLANNING INFORMATION QUALITY}

\author{
Fábio Favaretto ${ }^{1}$; Guilherme Ernani Vieira ${ }^{2}$ \\ ${ }^{1}$ PUC do Paraná - PUCPR - Curitiba - Brasil fabio.favaretto@pucpr.br \\ ${ }^{2}$ PUC do Paraná - PUCPR - Curitiba - Brasil gui.vieira@pucpr.br
}

\begin{abstract}
Resumo
O valor da informação é consenso atualmente, porém a maior parte das iniciativas das empresas valoriza apenas a tecnologia, e não o gerenciamento da informação. Uma forma de fazer este gerenciamento é conhecendo a qualidade da informação (QI). Este artigo faz um estudo com o objetivo de descrever a QI medida em situações de planejamento da produção. O método utilizado para a realização deste trabalho foi dividido em três etapas: revisão bibliográfica, aplicação de dois questionários e análise das respostas. Foram analisadas duas importantes etapas do planejamento da produção (planejamento mestre e planejamento detalhado da produção), para avaliação da qualidade das informações utilizadas por profissionais que participam na elaboração ou que dependem deste planejamento. A QI pode ser medida através de dimensões específicas, que apontam o quanto a informação atende às necessidades dos usuários ou consumidores de informação. Como resultados foram encontrados valores pouco acima das médias (intermediários) para a QI, mostrando oportunidades para melhorias.
\end{abstract}

Palavras-chave: qualidade da informação, dimensões de qualidade da informação, planejamento da produção.

\section{Introdução}

É comum ouvir que a informação é um bem da empresa. Isso remete a um tratamento diferenciado para a informação, indicando algo de valor. Para Rezende (2005), "mais do que nunca, a informação e seus respectivos sistemas desempenham funções fundamentais nas organizações, apresentando-se como recurso estratégico para projetar e gerir organizações de forma competitiva e inteligente". Beal (2004) destaca que, por outro lado, diferente de bens (ativos) comuns, a informação possui a característica de poder ser compartilhada indefinidamente.

Entretanto, os maiores esforços observados nas empresas não são relacionados ao tratamento, e sim ao processamento da informação, representado pela Tecnologia da Informação 
(TI). Na compra de um outro tipo qualquer de bem como, por exemplo, uma ferramenta, são observados diversos atributos deste produto, como custo, qualidade e durabilidade. Da mesma forma, a informação, que por analogia pode ser considerada um produto de informação (WANG, 1998), possui estes atributos, porém a qualidade da informação (QI) não é um atributo normalmente considerado em sua geração e utilização.

A QI tem impacto nos resultados de uma empresa, pois as decisões tomadas são baseadas em informações. Se a qualidade destas não for boa, a decisão pode ser comprometida. O uso de tecnologia avançada de processamento não garante a QI.

O processo de planejamento da produção é responsável por garantir que os produtos sejam entregues aos clientes de acordo com as necessidades destes. Várias decisões são tomadas durante este processo, utilizando e gerando diversas informações. Bazerman (2004) destaca a importância das informações no processo decisório, indicando diversas situações onde a decisão pode sofrer algum viés devido à forma como a informação é apresentada. Miranda (2004) apresenta a informação como um fator de produção, dado que o administrador precisa de informações, como os horários de trabalho, características do produto e outras. A forma de apresentação e a precisão são apenas alguns aspectos da QI.

O objetivo deste artigo é descrever a QI medida em situações de planejamento da produção. Foram entrevistados profissionais de diversas áreas, fornecendo um panorama geral sobre a qualidade de algumas informações geradas e utilizadas em atividades específicas de planejamento da produção.

A metodologia utilizada para o desenvolvimento é apresentada a seguir. Na seqüência são apresentados conceitos de QI e de planejamento da produção, e após são apresentados os questionários utilizados nesta pesquisa e os resultados obtidos. O artigo é finalizado com a análise dos resultados e as conclusões da pesquisa.

\section{Método}

Este trabalho apresenta um estudo descritivo. Segundo Hair Jr. et alli (2005), este tipo de pesquisa é estruturado para medir características, onde hipóteses derivadas da teoria guiam o processo de pesquisa e indicam o que precisa ser mensurado. Neste trabalho, será mensurada a qualidade da informação utilizada e gerada no planejamento da produção por um conjunto de empresas da Região Metropolitana de Curitiba.

O método utilizado para a realização deste trabalho foi dividido em três etapas: revisão bibliográfica, aplicação de dois questionários e análise das respostas. 
A revisão bibliográfica tem o objetivo de apresentar os principais conceitos de qualidade da informação e os conceitos básicos de planejamento da produção.

Para atingir o objetivo deste trabalho, foram elaborados dois questionários para analisar a qualidade da informação nas principais atividades de planejamento da produção. Para Hair Jr. et alli (2005), o survey realizado com o auxílio de questionários é uma das técnicas de coleta de dados para as pesquisas exploratórias.

Os questionários utilizados nesta pesquisa abrangem algumas informações utilizadas para realizar as atividades de planejamento da produção, assim como as informações geradas por estas atividades. Os questionários foram respondidos por profissionais graduados nas áreas de engenharia e administração de empresas médias e grandes que atuam em empresas de produção, alunos de um curso de pós-graduação lato sensu oferecido pela instituição onde os autores atuam. Estes profissionais são consumidores e geradores de informação. As respostas foram coletadas entre os meses de outubro e novembro de 2005.

Os questionários foram elaborados para se obter uma descrição da QI encontrada nas empresas onde os profissionais respondentes atuam, com base na avaliação realizada. Não foram testadas relações entre variáveis, isso será objeto de futuras pesquisas experimentais. As respostas dos questionários foram marcadas em uma escala numérica inteira de 1 a 5 , permitindo a tabulação dos resultados e elaboração de estatísticas descritivas.

A análise das respostas descreve a qualidade das informações com uma base objetiva (medidas), permitindo algumas conclusões e a elaboração de hipóteses e questões para futuras pesquisas.

\section{Conceitos de qualidade da informação}

A baixa qualidade da informação tem grandes impactos na efetividade geral de uma organização (WAND e WANG, 1996). Pesquisas realizadas na década de 90 do século passado revelaram que nos Estados Unidos, mais de 60\% das firmas de médio porte com vendas anuais de mais de 20 milhões de dólares tinham problemas com qualidade da informação. Nos arquivos de registros criminais nos EUA, de 50\% a $80 \%$ das informações são incompletas, ambíguas, ou sem acuracidade.

A base para o estudo da QI é o conceito de produto de informação (PI), uma analogia direta com produtos resultantes de processos de manufatura tradicionais. Estes produtos "físicos" são resultantes de um processo de produção onde matérias primas são processadas. O produto de informação é resultante do processamento de dados (brutos) por sistemas de informação. Essa analogia pode ser vista na Tabela 1. 
Tabela 1: Analogia entre sistemas de manufatura de produtos e de informações

\begin{tabular}{|l|c|c|}
\hline & Manufatura de produtos & Manufatura de informação \\
\hline Entrada & Matérias primas & Dados brutos \\
\hline Processo & Processo de manufatura & Sistema de informação \\
\hline Saída & Produto "físico" & Produto de informação \\
\hline Fonte: Adaptado de WANG et alli, (2000) \\
\hline
\end{tabular}

Assim como os produtos "físicos", a informação possui dimensões da qualidade associadas. Essas dimensões são agrupadas em categorias, conforme pode ser visto na Tabela 2. O senso comum aponta a acuracidade como sendo a própria QI, porém esta é apenas uma das dimensões.

Tabela 2: Categorias e dimensões da Qualidade da Informação.

\begin{tabular}{|l|l|}
\hline Categoria & Dimensão \\
\hline Intrínseca & Acuracidade, objetividade, credibilidade e reputação. \\
\hline Acessibilidade & Acessibilidade e segurança no acesso. \\
\hline Contextual & $\begin{array}{l}\text { Relevância, valor agregado, temporalidade, integridade e volume } \\
\text { de informação apropriado. }\end{array}$ \\
\hline Representação & $\begin{array}{l}\text { Interpretabilidade, facilidade de entendimento, representação } \\
\text { concisa, representação consistente e facilidade de manipulação. }\end{array}$ \\
\hline Fonte: Adaptado de WANG et alli, (2000) \\
\hline
\end{tabular}

Arndt e Langbein (2002) entendem qualidade de dados como "encontro consistente das "expectativas" dos consumidores de informação".

Conforme já exposto, a informação é tratada como um recurso, e por isso deve ser passível de mensuração, visto que os dados que são coletados, processados, acumulados e comunicados às empresas precisam ser mensurados de alguma forma (BEUREN, 2000). Assim, é possível medir a QI através da mensuração das dimensões apresentadas.

Guimarães e Évora (2004) afirmam que a informação é um recurso primordial para a tomada de decisão (no processo de produção). É inerente que esta informação tenha qualidade, no sentido de atender as necessidades do usuário. Neste trabalho serão consideradas apenas as dimensões acuracidade, credibilidade e temporalidade da QI. Estas dimensões foram escolhidas por representarem as principais características que uma informação relacionada ao planejamento da produção deve ter.

Para Shankar e Watts (2003), a avaliação de critérios de QI é uma tarefa difícil. A avaliação deveria ser tão precisa e prática quanto possível. Este é um conflito de objetivos e um compromisso difícil de alcançar. Uma avaliação imprecisa pode tanto resultar em uma informação recuperada de baixa qualidade ou levar a evitar informações de alta qualidade. Avaliação imparcial também pode resultar em uma avaliação imprecisa ou levar a uma avaliação indevida de tempo e custo. 
Conforme Wang e Strong (1996), três aproximações são utilizadas na literatura para estudar a qualidade de dados: uma intuitiva, uma teórica, e uma aproximação empírica.

A aproximação intuitiva é feita quando a seleção da qualidade dos atributos dos dados para qualquer estudo particular é baseada na experiência dos pesquisadores ou por entendimento intuitivo a respeito de quais atributos são "importantes". A maior parte dos estudos de qualidade de dados cai nesta categoria. Na literatura de informação de sistemas, qualidade de informação e a satisfação do usuário são as duas maiores dimensões para a avaliação do sucesso de um sistema de informação. Estas duas dimensões geralmente incluem alguns atributos da qualidade de dados, como a credibilidade, capacidade de tempo, precisão, confiabilidade, ocorrência, integridade (no sentido de estar completo), e relevância. Outros atributos como a acessibilidade e a interpretabilidade são também utilizados na literatura sobre qualidade de dados.

De acordo com Wang et alli. (2000), muitas iniciativas coorporativas, tais como o Businessto-Business, Gerenciamento Integrado de Cadeia de Suprimentos e o ERP (Enterprise Resources Planning), correm o risco de falhar se não forem consideradas a qualidade e a melhoria dos dados. A incerteza a respeito da qualidade e as perdas financeiras decorrentes da baixa qualidade dos dados e informações, bem como a dificuldade em gerar transformações e análises fora do padrão, têm sido motivos de verdadeiros pesadelos organizacionais.

\section{Conceitos básicos de planejamento da produção}

O planejamento da produção é um processo que deve responder às perguntas: o que, quanto, quando e como produzir. Independentemente do tipo da empresa, é necessário planejar como atender as necessidades dos clientes, entregando os produtos corretos no momento em que são necessários e dentro das especificações estabelecidas.

O início deste processo é através de informações sobre a demanda, que são as entradas. O processamento destas entradas mostra as necessidades de produção que ainda precisam ser planejadas.

Cabe ao planejamento da produção conciliar a produção de todos os tipos diferentes de produtos, que devem estar prontos em momentos planejados. Da mesma forma, o planejamento da produção é responsável por planejar a compra e disponibilização das matérias primas necessárias, também no momento correto.

Normalmente, o planejamento da produção é feito de forma hierárquica, sendo que a definição das necessidades dos clientes é o primeiro passo, chamado de gerenciamento da demanda. 
Conhecida a demanda, é feito inicialmente um planejamento de médio prazo para verificar as datas em que cada produto deve estar pronto. Esta etapa do planejamento é conhecida como planejamento mestre da produção, e serve como uma orientação geral para a produção.

De acordo com Corrêa e Corrêa (2004), o planejamento mestre da produção faz o cálculo das necessidades de produtos finais, indicando a quantidade e período de tempo em que deverão estar prontos. Para isso, são utilizados dados sobre a demanda dependente (quando o produto em questão faz parte - depende - de outro produto), sobre a demanda independente (que não depende de nenhum outro produto), dos pedidos em carteira e do nível de estoque dos produtos. Entretanto, esta etapa não detalha o planejamento da produção dos componentes de cada produto. Assim, o resultado do planejamento mestre alimenta uma próxima etapa que irá verificar estas necessidades.

Esta nova etapa é chamada de planejamento detalhado da produção (mais conhecida pela sigla MRP - Materials Requirements Planning). O MRP detalha então o plano mestre e determina os estoques intermediários e as datas de início e término da produção dos componentes. As etapas de planejamento mestre e detalhado são fundamentais para o planejamento de qualquer tipo de produção. Se este planejamento for falho, toda a empresa corre o risco de não se manter no negócio.

Para Corrêa e Corrêa (2004), o MRP é um cálculo das necessidades de materiais (componentes), que indica a quantidade destes, e as datas em que a produção deve ser iniciada e terminada. Para isso são necessários dados sobre a estrutura do produto (para conhecer os materiais que o compõe), o tempo de obtenção (entre o início e o término da produção) e o nível de estoque destes materiais.

Após a etapa de MRP, é realizada a etapa de programação da produção, que é um detalhamento do planejamento da execução de cada operação de produção de cada componente de cada produto a ser fabricado. Após esta etapa, ocorre a produção propriamente. Uma última etapa ocorre após a execução da produção, e faz o controle da produção, verificando se o planejamento foi cumprido, e caso não tenha sido, dá uma realimentação para que as outras etapas do planejamento sejam refeitas.

O processo de planejamento da produção é um grande integrador, pois precisa de informações de vários outros setores e processos da empresa, assim como as informações geradas no planejamento da produção são utilizadas por vários outros setores. Devido a esta importância em empresas de manufatura, as principais informações do planejamento da produção (planejamento mestre e MRP) serão analisadas nesta pesquisa. 


\section{Questionários e resultados da aplicação}

Foram aplicados dois questionários para se conhecer a qualidade da principais informações de entrada (dados) das etapas do planejamento da produção: um para a etapa de planejamento mestre e outro para a etapa de planejamento detalhado (MRP).

Para medir a QI nas três dimensões analisadas (confiabilidade, precisão e temporalidade), foram feitas questões específicas sobre cada uma das dimensões, para serem respondidas em uma escala inteira de 1 (péssima) a 5 (excelente).

No questionário específico sobre planejamento mestre da produção, foram feitas questões para cada dimensão das seguintes informações: previsão da demanda (independente), demanda dependente, pedidos em carteira e posição (nível) de estoque. Foi solicitado ao respondente para indicar em uma escala inteira de 1 (inexistente) a 5 (muito freqüente) a sua participação na elaboração do plano mestre, e em uma escala de 1 (inexistente) a 5 (muito grande) a dependência das suas atividades em relação ao plano mestre. Foram obtidas 56 respostas válidas e os resultados são apresentados na Tabela 3.

Tabela 3 - Resultados do questionário sobre a qualidade das informações relacionadas ao plano mestre de produção.

\begin{tabular}{|l|c|c|}
\hline \multicolumn{1}{|c|}{ Característica medida } & Média & Desvio padrão \\
\hline Confiabilidade da previsão da demanda & 3,30 & 0,97 \\
\hline Precisão da previsão da demanda & 3,14 & 0,86 \\
\hline Temporalidade da previsão da demanda & 3,29 & 0,95 \\
\hline Confiabilidade da demanda dependente & 3,29 & 0,82 \\
\hline Precisão da demanda dependente & 3,21 & 0,89 \\
\hline Temporalidade da demanda dependente & 3,21 & 0,89 \\
\hline Confiabilidade dos pedidos em carteira & 3,73 & 0,73 \\
\hline Precisão dos pedidos em carteira & 3,54 & 0,87 \\
\hline Temporalidade dos pedidos em carteira & 3,63 & 0,80 \\
\hline Confiabilidade do nível de estoque & 3,52 & 1,01 \\
\hline Precisão do nível de estoque & 3,23 & 1,11 \\
\hline Temporalidade do nível de estoque & 3,50 & 1,01 \\
\hline Participação na elaboração do plano mestre & 2,75 & 1,25 \\
\hline Dependência do plano mestre & 3,13 & 1,36 \\
\hline Fonte:Autores & & \\
\hline
\end{tabular}

As médias relacionadas às dimensões da QI ficaram entre 3,13 e 3,73, valores entre os conceitos médio e bom. Os valores da participação e dependência também ficaram próximos a esta faixa, porém o desvio padrão foi significativo, explicando uma variabilidade maior das avaliações. Outra característica, também com variabilidade diferenciada é a precisão.

No questionário específico sobre planejamento detalhado da produção (MRP), foram feitas questões para as dimensões de qualidade das seguintes informações: estrutura do produto, tempos 
de obtenção (lead-times) e posição (nível) de estoque. Foi solicitado ao respondente para indicar em uma escala inteira de 1 (inexistente) a 5 (muito freqüente) a sua participação na elaboração do MRP, e em uma escala de 1 (inexistente) a 5 (muito grande) a dependência das suas atividades em relação ao MRP. Foram obtidas 46 respostas válidas e os resultados são apresentados na Tabela 4.

Tabela 4 - Resultados do questionário sobre a qualidade das informações relacionadas ao planejamento detalhado da produção (MRP).

\begin{tabular}{|l|c|c|}
\hline \multicolumn{1}{|c|}{ Característica medida } & Média & Desvio padrão \\
\hline Confiabilidade da estrutura do produto & 3,63 & 0,80 \\
\hline Precisão da estrutura do produto & 3,43 & 0,78 \\
\hline Temporalidade da estrutura do produto & 3,43 & 0,93 \\
\hline Confiabilidade dos tempos de obtenção & 3,20 & 0,81 \\
\hline Precisão dos tempos de obtenção & 3,24 & 0,92 \\
\hline Temporalidade dos tempos de obtenção & 3,28 & 0,86 \\
\hline Confiabilidade do nível de estoque & 3,13 & 0,98 \\
\hline Precisão do nível de estoque & 2,98 & 1,04 \\
\hline Temporalidade do nível de estoque & 3,26 & 1,06 \\
\hline Participação na elaboração do planejamento detalhado & 2,35 & 1,20 \\
\hline Dependência do planejamento detalhado & 3,09 & 1,31 \\
\hline Fonte:Autores & & \\
\hline
\end{tabular}

As médias relacionadas às dimensões da QI ficaram entre 2,98 e 3,63, valores aproximadamente entre os conceitos médio e bom. O valor da dependência também ficou próximo a esta faixa, porém o desvio padrão foi significativo. O valor da participação ficou sensivelmente baixo $(2,35)$, próximo ao conceito pequena e também com desvio padrão significativo. Estas duas características apresentaram padrões relativamente parecidos para ambas etapas do planejamento analisadas.

$\mathrm{Na}$ Tabela 5 são apresentadas as médias e desvios para os valores globais de todas as respostas para cada característica medida.

Tabela 5 - Médias e desvios globais para as características medidas.

\begin{tabular}{|l|c|c|}
\hline \multicolumn{1}{|c|}{ Característica medida } & Média & Desvio padrão \\
\hline Confiabilidade & 3,41 & 0,90 \\
\hline Precisão & 3,26 & 0,94 \\
\hline Temporalidade & 3,38 & 0,93 \\
\hline Fonte:Autores
\end{tabular}

Estas médias indicam que, de uma forma geral, a QI percebida pelos participantes é pouco maior que a média. 


\section{Análise dos resultados e conclusões}

Inicialmente, pode-se afirmar que as dimensões de QI analisadas tiveram valores pouco acima da média, porém considerando os desvios padrões, podemos aproximar os valores para a média. Isso significa que os profissionais que responderam ao questionário não consideram as informações que utilizam nem boas nem ruins. Não houve diferenças significativas entre os conjuntos de informações ou entre as dimensões analisadas.

Considerando-se cada etapa do planejamento, nota-se uma variabilidade (através do desvio padrão) um pouco maior (cerca de 10\%) nas respostas relativas ao MRP. Para esta etapa do planejamento, a participação dos entrevistados é relativamente menor (média de 2,35), o que pode sugerir que a falta de participação dos entrevistados na elaboração do MRP pode levar a uma maior variabilidade na percepção da QI.

Em uma análise mais detalhada foram feitas as correlações entre todas as respostas obtidas. Essa análise não apresentou correlações significativas entre a dependência e participação com as informações analisadas para cada etapa do planejamento da produção. Com isso, pode-se afirmar que não necessariamente o indivíduo que marcou valores altos ou baixos para a QI tem grande ou pequena participação na elaboração das etapas de planejamento analisadas. O mesmo ocorre com a dependência das atividades realizadas em relação ao planejamento.

Em ambas as etapas do planejamento da produção, a proporção de entrevistados que participa na elaboração é relativamente pequena (médias inferiores a 3), sendo que a dependência dos entrevistados em relação ao MRP e Plano Mestre é ligeiramente maior. Isso pode estar encobrindo algum tipo de correlação que não foi avaliada nesta pesquisa, e pode sugerir a hipótese de que as percepções daqueles que participam na elaboração destas etapas do planejamento é diferente daqueles que dependem destas etapas. Esta hipótese será avaliada em pesquisas futuras.

Conclui-se que as informações utilizadas pelos indivíduos que participaram desta pesquisa possuem oportunidades para melhorias, o que certamente pode melhorar a qualidade do planejamento da produção e consequentemente o desempenho da empresa. Vale ressaltar que estas conclusões são válidas para a amostra analisada, e não podem ser generalizadas para todos os ambientes de produção.

Como continuidade deste trabalho serão feitas pesquisas experimentais para investigar se a relação de fatores como a utilização de sistemas de informação e a forma de obtenção das informações afetam a qualidade da informação resultante. 


\title{
Agradecimento
}

O desenvolvimento desta pesquisa teve apoio financeiro do Conselho Nacional de Desenvolvimento Científico e Tecnológico (CNPq) através do Edital Universal.

\begin{abstract}
It is common to say that information is a precious asset but most of enterprises initiatives are in technology direction despite of information management. One way to do this management is to know information quality (IQ). This article shows a study with main goal in describe measured information quality in production planning situations. The research methodology used in this work has three steps: concepts and literature review, two specific questionnaires application and results analysis. There were analyzed two important production planning activities: master plan schedule and materials requirements planning to verify used and generated information quality. IQ can be measured using specific dimensions that points out if users' needs are supported. Medium IQ values were found as a result giving some improve opportunities.
\end{abstract}

Key words: information quality, information quality dimensions, production planning.

\section{Referências}

ARNDT Dirk; LANGBEIN Norman. Data quality in the context of customer segmentation. Proceedings of the Seventh MIT International Conference on Information Quality (ICIQ-02), 2002.

BAZERMAN, M. H. Processo decisório. Tradução da quinta edição, Elsevier, Rio de Janeiro, 2004.

BEAL, A. Gestão estratégica da informação. Editora Atlas, São Paulo, 2004.

BEUREN, I. M. Gerenciamento da informação. Segunda edição. Editora Atlas, São Paulo, 2000.

CORRÊA, H. L.; CORRÊA, C. L. Administração de produção e operações. Editora Atlas, São Paulo, 2004.

GUIMARÃES, E. M. P; ÉVORA, Y. D. M. Sistema de informação: instrumento para tomada de decisão no exercício da gerência. Ciência da informação, Volume 33, Número 1, Janeiro/Abril, 2004.

HAIR JR., J. F.; BABIN, B.; MONEY, A. H.; SAMOUEL, P. Fundamentos de métodos de pesquisa em administração. Bookman, Porto Alegre, 2005.

MIRANDA, S. V. Identificando competências informacionais. Ciência da Informação, Volume 33, Número 2, Maio/Agosto, 2004.

REZENDE, D. A. Sistemas de informações organizacionais. Editora Atlas, São Paulo, 2005.

SHANKAR, G. \& WATTS S. A relevant, believable approach for data quality assessment. Proceedings of the Eighth MIT International Conference on Information Quality (ICIQ-03), 2003.

WAND, Yair e WANG, Richard Y. Anchoring Data Quality Dimensions in Ontological Foundations, Communications of the ACM, Número 39, 1996.

WANG, Richard Y. A Product Perspective on Total Data Quality Management. Communications of the ACM, Vol. $41, \mathrm{n}^{\mathrm{o}} 2,1998$.

WANG, R. Y.; ZIAD, M.; LEE, Y. W. Data quality. Kluwer Academic Publishers, 2000. 
WANG, Richard; STRONG, Diane M. Beyond Accuracy: What Data Quality means to Data Consumers. Journal of Management Information Systems, Volume 12, Número 4, 1996.

\section{Autores:}

Nome completo: Fábio Favaretto

Filiação institucional: Pontifícia Universidade Católica do Paraná

Departamento: Programa de Pós-Graduação em Engenharia de Produção e Sistemas

Função ou cargo ocupado: Professor adjunto

Endereço completo para correspondência (bairro, cidade, estado, país e CEP): Parque Tecnológico

- Bloco 3 - Segundo andar. Rua Imaculada Conceição, 1155 - Prado Velho, Curitiba - PR - CEP 80215-901

Telefones para contato: 41-3271-1344

e-mail: fabio.favaretto@pucpr.br

\section{Nome completo: Guilherme Ernani Vieira}

Filiação institucional: Pontifícia Universidade Católica do Paraná

Departamento: Programa de Pós-Graduação em Engenharia de Produção e Sistemas

Função ou cargo ocupado: Professor adjunto

Endereço completo para correspondência (bairro, cidade, estado, país e CEP): Parque Tecnológico

- Bloco 3 - Segundo andar. Rua Imaculada Conceição, 1155 - Prado Velho, Curitiba - PR - CEP 80215-901

Telefones para contato: 41-3271-1344

e-mail: gui.vieira@pucpr.br

Recebido para publicação em: 05/03/06

Aceito para publicação em: 10/05/07 